\title{
La voie du goût
}

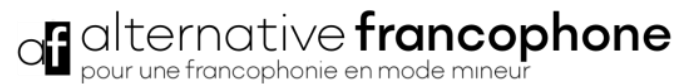

\section{https://doi.org/10.1215/08992363-10.29173/af29422}

\section{(@) $\odot \Theta$}

\section{Marc Kober}

marc.kober@univ-paris13.fr

Université Sorbonne-Paris Nord

Résumé. L'intégration d'éléments qui renvoient aux singularités de la gastronomie et des pratiques culinaires du Japon est un phénomène assez courant que ce soit dans les romans, les essais, les guides, les albums dessinés, les productions numériques, ou les films. Ces insertions ont un rôle direct d'information sur ce pays (ou sur ses excroissances mondialisées) mais aussi un rôle de consolidation d'un imaginaire de la culture japonaise. Cette présence sera interrogée ici à la fois sous l'angle d'une production esthétique et sensorielle (la cuisine élevée au rang des beaux-arts), et sous l'angle d'une construction herméneutique.

Mots clés : Japon; cuisine; gastronomie; littérature

Abstract. The integration of elements that refer to the peculiarities of Japanese gastronomy and culinary practices is a fairly common phenomenon in novels, essays, guides, comic books, digital productions, and films. These inserts have a direct informative role on this country (or on its globalized outgrowths) but also a role of consolidation of an imaginary of the Japanese culture. This presence will be questioned here both from the point of view of an aesthetic and sensory production (the kitchen elevated to the rank of fine arts), and from the point of view of a hermeneutic construction.

Keywords: Japan; cooking; gastronomy; literature

$\mathrm{P}$ armi les objets transversaux qui renouvellent l'approche française du Japon figurent la gastronomie et l'art culinaire. Ce transfert entre deux cultures est une modalité presque naturelle pour le visiteur ou le résident. Il faut remonter au gastronome Rosanjin Kitaôji (1883-1959), ou à certaines pages de Tanizaki Jun'ichirô (1886-1965), pour comprendre comment des éléments non littéraires (le guide de voyage, le livre de recettes, la carte de restaurant) peuvent être élevés au rang de création littéraire. La gastronomie n'est pas une simple mise en bouche, mais une façon d'approfondir une familiarité. Le premier plan de l'art culinaire serait esthétique. Cet art serait encore producteur de plaisir comme élément central dans une perspective hédoniste. Il serait enfin un stimulant cognitif de premier 
ordre. À cet égard, l'art culinaire est un code qui appelle son « décodeur », une clé métonymique. L'expérience gastronomique est servie par une grande variété de moyens (du simple haïku au roman), et le dialogue culturel se renouvelle par l'approche visuelle permise par la production graphique, cinématographique, ou numérique.

\section{UNE IMMERSION GUSTATIVE PAR LES MOTS}

\section{Journalisme gonzo ${ }^{1}$ et narration non fictionnelle}

Dans un premier temps, une dimension documentaire est impliquée par l'évocation gastronomique, dans l'esprit du new journalism ${ }^{2}$ avec immersion totale, voire une pratique réelle de consommation répondant à des besoins parfois compulsifs de satisfaire un besoin primaire, voire d'y puiser une source d'inspiration, comme dans le reportage dit «gonzo ». L'étymologie de ce mot renvoie à une absorption d'alcool excessive. En effet, ce genre de reportages supposait de la part du journaliste une immersion quasi ethnographique dans l'univers qu'il souhaitait présenter aux lecteurs, en partageant le mode de vie des personnes étudiées. Changer radicalement son mode de vie devenait une condition nécessaire. Notre hypothèse sera que l'expérience gastronomique japonaise appelle ce genre d'immersion. Et l'un des charmes de L'Empire des signes est que Roland Barthes évoque minutieusement des expériences culinaires. Sans faire du sémiologue français un reporter gonzo, il est probable que ses voyages successifs au Japon ont été vécus sur le mode d'une immersion dans les « signes » du Japon, où figure en bonne part l'écriture des mets. Il est « l'un des premiers à se livrer à une investigation passionnée de la vie quotidienne $\gg$ (Dubois 80).

\section{L'expérience de l'alcool et des mets qui l'accompagnent}

À propos du vin ou du saké, la relation culturelle fonctionne dans les deux sens avec la recherche d'une équivalence gustative. Nombre d'ouvrages renvoient à une littérature non fictionnelle, où une expérience immersive se manifeste. La littérature de non-fiction semble prendre le dessus sur la fiction pure. Les faits dominent le texte qui, pourtant, suit les techniques narratives les plus littéraires en se situant à l'intersection d'une enquête ethnographique, d'un témoignage subjectif et d'une narration très peu fictionnelle. Si l'on part du degré zéro, nous pouvons faire appel aux manuels (handbooks) pour l'amateur de saké, ou de bonne cuisine, sans parler d'autres domaines. Ces manuels sont éclairants pour comprendre les attentes d'un public étranger, la façon dont les auteurs se mettent dans l'esprit japonais, ou partent du point de vue japonais pour imposer au lecteur étranger une traduction. Jake Adelstein, un journaliste

\footnotetext{
${ }^{1}$ Le gonzo serait, selon le Webster's new world dictionnary en 1979, un synonyme de « bizarre, unrestrained, extravagant, specially designating a style of personal journalism so characterized » (Woo). Une autre définition serait une méthode d'enquête ultra subjective, un journalisme « undercover » une « opération d'hybridation » (Binet) entre forme romanesque et reportage, avec une dimension autobiographique et des anecdotes hautes en couleur.

${ }^{2}$ Le nouveau journalisme, ou saturation report, personal narrative, est un journalisme d'investigation au service de la fiction, un style de reportage à dimension esthétique. Il est encore présenté comme un « journalisme alternatif » (Kaganski).
}

Alternative francophone

https://journals.library.ualberta.ca/af/index.php/af 
américain basé au Japon, évoque dans l'un de ses ouvrages le classement des manuels les plus achetés au Japon sur le site Amazon :

Aujourd'hui l'obsession des Japonais pour la bonne manière de faire est toujours aussi vive. Il y a quelques années, l'expression manual ningen (les hommes manuels) était à la mode pour désigner une génération de jeunes Japonais incapables de toute indépendance d'esprit. L'expression fait aujourd'hui partie du langage courant, et désigne celui qui ne peut pas s'empêcher de suivre les instructions et est incapable de sortir des clous (Adelstein 93-4).

Toutefois, malgré l'engouement des Japonais pour la gastronomie, aucun manuel portant sur ce thème ne figurait dans les dix premières ventes. En revanche, on trouvera fréquemment au rayon « langues étrangères » des librairies japonaises de nombreux manuels ou guides censés aider l'étranger résidant au Japon à se cultiver dans un domaine réputé difficile comme le saké. Le manuel de John Gauntner est remarquablement clair puisqu'il est composé d'une centaine de fiches qui indiquent le nom du saké en kanjis, puis en caractères romains, la qualité et l'origine, avec une reproduction de chaque étiquette, un tableau avec six indications précises, puis une appréciation du goût extrêmement fouillée, dans l'esprit de celles des sommeliers ou des tastevins, ou dans celui des appréciations qu'on peut lire sur les bouteilles de vin en France. Cette série de fiches est suivie par une liste de bonnes adresses où boire du saké à Tokyo, puis par un glossaire. Les pubs à saké sont indiqués avec des plans à partir de la gare la plus proche et tous les détails pratiques qui permettront au lecteur de vivre, s'il le souhaite, une véritable expérience. La dimension littéraire du texte se réfugie dans l'évocation métaphorique du goût du saké comme il est d'usage pour vanter les bonnes bouteilles de vin ou d'alcool en France. Ce manuel est donc une invitation à rencontrer le saké, mais à condition de bien lire l'introduction qui comporte un schéma sur le mode de fabrication en une page, un chapitre sur les ingrédients, ou un autre sur l'art de goûter le saké. Il faut donc lire quatre-vingts pages explicatives avant d'entrer dans la liste des brasseurs. Le guide de Philip Harper est d'un format plus maniable, mais il comporte des éléments supplémentaires, tel l'historique de la fabrication d'alcool au Japon depuis les origines, une carte de l'archipel pour le buveur au Japon, ou dans la jaquette, une rubrique pratique, «deciphering a sake label» (comment lire l'étiquette d'un saké). Avant la liste des brasseurs de saké, on trouve sur soixante-cinq pages serrées à peu près les mêmes éléments techniques et même la description-type d'une étiquette pour ne pas se tromper lors de l'achat. Là encore, il s'agit d'initier et de rendre en théorie l'étranger autonome dans un domaine complexe, mais l'effet peut être aussi bien paralysant ou énigmatique. Il semblerait que les auteurs de ces manuels aient repris ici les caractéristiques de l'approche extrêmement précise et sérieuse des manuels japonais. Par ailleurs, il faut savoir qu'une multitude de guides et de manuels existent au Japon pour expliquer et décrire par exemple les habitudes alimentaires des Français et les produits qu'ils préfèrent consommer. Ces manuels existent pour chaque gastronomie européenne, et développent bien d'autres sujets. D'une manière sérialisée, on pourra trouver des ouvrages sur les pâtisseries, les vins, les fromages ou le pain. Ces ouvrages s'agrémentent de photographies ou de dessins avec de multiples légendes et transcriptions en syllabaire katakana pour restituer la prononciation des mots. Ce qui fait que ces ouvrages en japonais, pris dans leur ensemble, constituent une véritable encyclopédie du monde, une carte géante qui couvre bien des domaines. L'existence des manuels du type de ceux évoqués par rapport au saké est donc une réplique homologique par des auteurs américains à cet effort de traduction et de représentation des pratiques étrangères dans le domaine des habitudes du quotidien.

Le manga de Taniguchi Jirô, Le Gourmet Solitaire, est précieux par la distinction qu'il établit entre les Japonais qui boivent de l'alcool et ceux qui n'en consomment pas. En effet, l'expérience culinaire au Japon est vraiment tributaire de cette distinction. Nous reproduisons la note du chapitre 18, le dernier, qui abandonne le héros solitaire à cette réflexion désabusée : «Ma foi, je resterai toujours un Japonais qui ne 
boit pas » (Taniguchi 184). Autrement dit, un Japonais qui ne sera jamais tout à fait à l'aise dans un izakaya, ces petits restaurants où les plats sont forcément accompagnés par de l'alcool :

La nourriture au Japon se range en deux grandes catégories, selon sa fonction : celle que l'on prend pour se restaurer et celle que l'on prend pour accompagner l'alcool. Plus précisément, sans riz, les mets s'articulent autour de l'alcool qu'on boit en grignotant. Un gourmet qui ne boit pas d'alcool est une sorte de loup solitaire. L'empêcher de prendre du riz et de faire un repas licite, quand tout le monde autour de lui boit, c'est comme lui rappeler agressivement son « orientation gustative ». (Taniguchi 175)

Les guides que nous venons d'évoquer traitent de façon assez indépendante du problème du saké, et n'éclairent pas la relation très codée qui s'établit entre alcool, relations sociales et professionnelles, et prise du repas. D'autres ouvrages apportent une réponse plus synthétique. Ce sont différents types de cuisine et différentes manières de boire et de manger qui sont présentés au lecteur dans certains guides qui entendent « dépayser » la pensée du Japon par les Occidentaux pour lutter contre les clichés qui surchargent son approche. C'est le cas avec Le Japon des Japonais. Dans un chapitre de ce guide, «L'appétit joyeux », les auteurs insistent sur le sens de la fête des Japonais et sur la reconnaissance de la gourmandise (kuidoraku) comme une expérience extrêmement importante. Au-delà de la simple présentation, la cuisine serait une « [...] nouvelle voie, celle du kuidoraku qui conjugue sociabilité et plaisir des sens » (Pons et al. 132). Les auteurs en veulent pour preuve le choix infini des restaurants à Tokyo, « une des villes les plus cosmopolites de la planète et une des capitales de la gastronomie pour la variété et la qualité des cuisines que l'on y sert »(130), mais également l'importance télévisuelle du thème de la cuisine, avec de nombreuses émissions sur les recettes ou les restaurants, l'abondance en librairie des magazines ou livres de cuisine, et même des bandes dessinées relatives aux aliments ou aux recettes. Les auteurs insistent sur l'importance de la dérive nocturne, dans un monde qui est surtout celui des mets qui accompagnent l'alcool, suivant un véritable « cheminement gustatif » (131). Quelques auteurs français ont restitué leur expérience gastronomique au Japon de manière assez minutieuse, l'un en tant que chroniqueur gastronomique, l'autre en tant que cuisinier français en tournée au Japon. Parmi eux, nous évoquerons l'immersion fascinante de Laurent Feneau, chroniqueur gastronomique, dans l'univers des sakés, ou celle de Philippe Delacourcelle, un cuisinier français venu s'intéresser à la cuisine japonaise dès 1979 et sur plusieurs décennies.

\section{La voie du saké}

Le petit ouvrage de Laurent Feneau est précieux par l'identification convaincante qu'il opère entre découverte du saké et «plaisir presque constitutif d'un séjour au Japon » (13), une authentique «voie du saké » (14). Auteur d'un livre de chroniques culinaires, Paris-Tokyo : dialogue des sens, Laurent Feneau privilégie les allers-retours entre les deux cultures, et tisse une série d'analogies entre vin et saké d'une part, entre gustatif et dimension onirique ou visionnaire d'autre part. Il se situe à la fois pleinement dans une dimension cognitive et dans une approche sensible et imaginaire, sans jamais tomber dans le piège de l'unité ou du type pur (défaut des nihonjinron, essais sur les Japonais), d'une conception nationaliste de l'identité culturelle). Ainsi, l'auteur préfère se situer dans des «non-lieux » (Augé), tels que les ascenseurs, les bibliothèques, les carlingues d'avion ou dans des lieux hybrides (parcs « japonais » de Paris) pour déguster des sakés typiques. Plus sûrement, l'auteur se situe entre Paris et divers lieux du Japon, ce qui déjoue l'approche du seul « voyage au Japon ». Ainsi, le livre s'ouvre avec l'évocation d'Akasaka, quartier de la capitale japonaise, mais entre une chambre d'hôtel neutre et un konbini (convenience store), pour la dégustation d'un saké qui n'a rien de traditionnel, suivant l'idée qu'il faut,

Alternative francophone

https://journals.library.ualberta.ca/af/index.php/af 
dans ce domaine, s'ouvrir à l'inattendu. Le second chapitre porte sur l'analogie entre vin et saké, et relate une dégustation à Paris d'un type de saké dit « d'éducation », où l'auteur reconnaît notamment l'acidité de certains Sauvignons et utilise pour le saké un lexique propre à la description des vins français. Les vins de terroir seraient l'équivalent des sakés de pays. Bref, il s'agit de se rattacher à des domaines connus pour assimiler l'inconnu, « s'éloigner de la sphère cognitive spécifique au domaine viticole afin de pénétrer véritablement le domaine complexe des saveurs propres aux vins de riz » (Feneau 26). C'est donc une méthodologie qui est proposée, pour se déshabituer des connaissances déjà acquises tout en s'appuyant sur elles comme un tremplin. Pour le reste, le goût du saké est le lieu d'une réminiscence, le moyen d'une évasion imaginative et mémorielle. Au-delà de l'aspect cognitif, c'est l'imagination sensorielle des atmosphères japonaises qui est permise par le vin de riz. Après quelques généralités sur le développement social (izakayas) et esthétique du saké (les flacons, ou tokkuri), l'auteur insiste sur la nature poétique du saké : «Tout vin de riz est un poème ou plutôt un récit » (39). En effet, sa saveur est évocatrice du sol, de l'eau, du riz et des hommes. Ainsi, les rizières sont visitées avant la brasserie pour un saké de terroir, mais la visite émerveillée du laboratoire de la brasserie est non moins révélatrice. Pourtant, ce livre ne se réduit pas à des visites. Il se déroule tout autant dans la nostalgie, à distance, et dans l'élaboration d'expériences analogiques qui déplacent le Japon en France, et cherchent des solutions à l'absence de grille de lecture pour le Japon, et pour le vin de riz en particulier. Le chapitre « bois » (au sens forestier) est une grande page de la gastronomie franco-japonaise, mais surtout la démonstration vivante de l'autonomie du sujet gastronome qui emporte avec lui le goût et l'expérience sensorielle du Japon n'importe où ailleurs, dans les entre-deux franco-japonais. L'avant-dernier chapitre révèle que certains sakés ginjô, des sakés sélectionnés pour leur excellence, permettent de s'affranchir des limites terrestres, où que l'on soit.

\section{L'addiction aux sushis}

Philippe Delacourcelle intègre la découverte de la culture japonaise à une activité professionnelle intense de cuisinier et à des échanges humains recherchés : «[...] et le séjour qui ne devait être qu'une parenthèse s'est transformé en cinq années d'immersion complète dans d'autres cultures et d'autres gastronomies » (13). Cette immersion d'une dizaine d'années est condensée en chapitres qui présentent l'expérience japonaise sous l'angle gastronomique. Et c'est l'atmosphère qui est restituée d'une manière intimiste, que ce soit dans les maisons privées ou les établissements spécialisés dans tel ou tel type de cuisine : «La buée diffusée par la machine à riz et celle de la soupe "misoshiru", en permanence prête à être servie sur le coin du fourneau, embaume invariablement l'air ambiant. L'éclairage est très japonais. D'énormes plafonniers actionnés par un cordon diffusent une lumière froide et trônent au milieu des pièces » (26). Ce texte parvient à envelopper le lecteur dans l'atmosphère japonaise sans jamais tomber dans une description convenue, et l'approche de l'intérieur des restaurants où le cuisinier français a pu travailler est vraiment précise et instructive. En décrivant un premier restaurant de sushis (sushi-ya) à Sendai, il tente une définition de l'approche japonaise par « [...] une quête de la magnificence de la matière première alliée à une présentation unique [...] » (31). Le chapitre concernant le marché en gros des poissons à Tsukiji magnifie encore cette fraîcheur et le souci de cuisiner des produits du terroir. À Aomori, ville du nord du Japon, l'auteur s'étonne de rencontrer une connivence bien établie entre les deux gastronomies française et japonaise : « Certains d'entre eux connaissent la France, d'autres n'y sont jamais allés, mais tous me surprennent par leur connaissance de la gastronomie française et des produits qui la constituent » (56). En évoquant différentes expériences presque inévitables, tels le voyage en shinkansen avec sa restauration à bord et dans les gares, la street food, les konbini (convenient stores) et leur offre pléthorique en aliments, les ryôkan (auberges traditionnelles), les nomiya, versions plus populaires des izakaya, les deux servant essentiellement de l'alcool avec des plats d'accompagnement, et les bars, où se mêlent alcool et mets d'accompagnement, la cuisine raffinée de Kyoto, le riz, la friture, ou les sushis, il trouve 
quelques clés explicatives de la différence japonaise, en particulier par la spécialisation des établissements qui est un gage de qualité :

Il me semble que la grande différence entre la cuisine japonaise et la cuisine française se situe dans la spécialisation des restaurants. La restauration au Japon est l'affaire de petites unités où le patron s'efforce de cuisiner un nombre réduit de plats, reproduits toute une vie durant et repris par les descendants, de manière à faire perdurer l'entreprise familiale. (Delacourcelle 97)

À l'inverse, « le cuisinier français est un généraliste » (97). Avec un résultat très positif pour la méthode japonaise. Trois cuisines retiennent l'attention de l'auteur, et ce sont à peu près les mêmes que celles retenues par Roland Barthes : les sushis, la tempura (friture), et la cuisine traditionnelle, dite kaiseki. Si la tempura est « l'art de cristalliser un produit » par un bain de friture qui transforme tout en « dentelle croustillante » (100), l'auteur montre combien cet art dépend de l'authenticité et de la fraîcheur des produits. La cuisine kaiseki est une représentation du Japon traditionnel, et, comme nous le verrons avec les réflexions de Ryôko Sekiguchi ${ }^{3}$, une façon de «mettre en valeur le temps présent » (111) où « l'ordre de la nature est la règle majeure »(111), autrement dit la succession des saisons recréée par la vaisselle, la décoration et le choix des aliments. L'évocation à cet égard d'un souper dans une auberge des environs de Kyoto est immersive et repose sur un enveloppement olfactif, sur un contact entre l'épiderme nu sous le peignoir yukata et les saveurs locales ou fusionnelles qui sont servies. Mais finalement, les pages les plus vibrantes sont écrites sur le sushi, « ambassadeur de la culture japonaise à travers le monde », et vecteur d'une équivalence généralisée entre « cuisine japonaise » et «poisson cru » (88). Il en décrit les origines et les différentes saveurs avec précision, mais au fond, il reconnaît qu'il s'agit d'une véritable « addiction »: « pour moi, c'est sans doute le summum du plaisir gustatif » (105). Où l'on retrouve la nécessité pour bien écrire sur la cuisine japonaise de s'y abandonner totalement jusqu'à une forme de monomanie. Cependant, ce choix est justifié par la subtilité de l'art du maître sushi, contraint d'étudier dix années avant de porter ce titre, par l'importance des produits mis en œuvre, poisson, riz, eau, nori (algue en feuille séchée), et par l'accord établi entre saisons et variété des sushis préparés. Comme un hommage intemporel, l'auteur évoque un menu particulier consommé dans la sushi-ya de Monsieur Kidoguchi, un rituel entre confrères, car « la cuisine est une clé magique, elle devient le lien d'une confrérie que l'on ne soupçonne pas en France » (131).

\section{Kenji Fujimoto et les meilleurs sushis du monde}

Ce maître sushi n'aurait sans doute pas fait parler de lui sans un concours de circonstances qui lui permit de devenir le cuisinier et l'ami du général Kim Jong-il, père de l'actuel dirigeant de la Corée du Nord. Cette chronique d'une douzaine d'années éclaire bien la perception de la cuisine japonaise dans un pays de culture voisine. Fujimoto est démarché pour ouvrir au départ un restaurant de sushis à Pyongyang, mais il devient bien mieux : un intime du dirigeant nord-coréen. On apprend que ce dernier adore les sushis préparés avec la poitrine de thon (toro) et un riz japonais. Une relation amicale s'établit d'abord autour d'une passion pour les sushis, puis sur différents plats typiquement japonais, comme les tempura soba ou le tanuki udon. Certains aspects de la cuisine japonaise semblent propices à une diplomatie culinaire qui ne dit pas son nom. Ce cuisinier semble motivé par le bonheur de préparer des sushis devant

\footnotetext{
${ }^{3}$ Le petit essai intitulé Nagori suit une réflexion sur les saisons des produits et des cuisines au Japon et ailleurs en insistant sur la sensibilité particulière des Japonais pour les saisons, avec une préférence pour l'automne, saison par excellence du nagori.
}

Alternative francophone

https://journals.library.ualberta.ca/af/index.php/af 
son éminent employeur, au point de s'engager à ne pas retourner au Japon pendant cinq années. Il divorce et oublie sa famille, épouse une Nord-coréenne, et continue à développer des spécialités japonaises pour les Nord-coréens, comme l'ikizukuri (poisson découpé vivant, ce qui permet de conserver les nerfs actifs dans la chair). La passion du dirigeant pour le sushi de poisson gras (thon) est telle qu'il oublie même l'interdiction formulée par son médecin d'en consommer, tandis que son cuisinier est si enthousiaste pour son statut de cuisinier d'élite qu'il se trouve de fait prisonnier et sous surveillance, tributaire de l'amitié d'un homme et de son épouse. Fujimoto décrit minutieusement le palais exceptionnel du général, ses goûts délicats assez contraires à la cuisine très pimentée et épicée des Coréens. Il n'aime pas le kimchi ou le wasabi, et préfère la saveur sucrée du sukiyaki, les sômen, ou la friture de légumes en tempura. Parmi de très nombreuses anecdotes qui émaillent son récit, Fujimoto raconte comment le général remarqua en 1992 le goût différent des sushis, et comment il put vérifier effectivement qu'il avait utilisé dix grammes de sucre en moins dans l'assaisonnement du riz avec vinaigre, sel et sucre. La passion gastronomique du maître de la Corée du Nord était telle que Fujimoto partait acheter par avion des quantités très importantes de thon ou d'encornet congelés, ou des pâtisseries de Ginza. Son goût immodéré pour les sushis rendait leur présence régulière plusieurs fois par semaine au menu du dirigeant, qui « adorait le goût délicat de la cuisine japonaise » (Fujimoto 183), en particulier le poisson cru, les cèpes ou des onigiri raffinés. Après deux années d'interruption, le cuisinier japonais revint préparer des sushis et le général se serait alors exclamé : « les sushis de Fujimoto sont les meilleurs du Japon, donc les meilleurs du monde » (186), ce qui résume la nature de cette amitié gastronomique. En 2001, le cuisinier, qui a pris conscience de la fragilité de sa situation, décide de s'enfuir, et ce seront les sushis qui le sauveront. En effet, il montre au dirigeant une vidéo du New Dotch Cooking Show, une série diffusée entre 1997 et 2005 par la Yomiyuri Telecasting Corporation, qui présentait des recettes exécutées avec les meilleurs ingrédients du monde. L'émission est consacrée à la préparation d'un unidon, bol de riz très richement garni de langues de corail d'oursins venus du Hokkaïdo. Et comme le prévoyait le rusé Fujimoto, Kim Jong-il est enthousiaste au point de lui donner carte blanche pour aller en chercher le mois suivant. Fujimoto ne revit jamais son cher dirigeant, et les sushis, qui avaient servi de sésame pour son entrée, furent le moyen inespéré de fuir la surveillance policière. Cette histoire démontre combien l'appartenance au milieu des cuisiniers favorise la traversée des frontières et met en contact des cultures officiellement contradictoires.

\section{Le Japon à Paris}

L'existence dans la capitale française d'une communauté japonaise relativement importante (entre 10.000 et 20.000 personnes) a favorisé l'implantation de commerces où l'alimentaire et la restauration occupent une place de choix. Ces restaurants sont répertoriés, ainsi que diverses informations sur la vie au Japon et en France, à l'usage des Japonais, mais surtout des Français, dans des journaux et magazines gratuits comme «Zoom» ou « Ovni », distribués à l'entrée des restaurants nippons. Mais certains guides commencent à proposer un itinéraire japonais, comme le Guide du Japon à Paris. L'auteur est une Japonaise venue travailler à Paris, ce qui est aussi la situation de J.P. Nishi, auteur de mangas dont la saga parisienne est relatée avec humour en deux volumes traduits en français. Avec la première partie " gastronomie », soixante et une pages sur cent quarante et une au total concernent les restaurants et autres bistrots ou salons de thé, soit presque la moitié du Guide du Japon à Paris. Cette longue énumération des restaurants par catégories, avec un descriptif synthétique typique des guides gastronomiques, est suivie par les sections «shopping, mode et déco », « beauté et bien-être » et enfin «pratique » qui donne des indications pratiques pour suivre une activité culturelle ou des cours de langue à Paris. Enfin, le guide propose quatre itinéraires types dans divers quartiers de la capitale, quatre « journées japonaises » sans se déplacer en quelque sorte. L’idée est de découvrir le Japon sans quitter le sol parisien. L'ensemble des lieux et des personnes évoqués constitue - mieux qu'un parc d'attractions avec des répliques à échelle réduite des monuments européens - un espace japonais disséminé dans 
l'espace parisien. Il s'agit de percevoir la proximité d'une autre culture à travers ses différences, mais aussi ses moments fusionnels, puisque nombre de restaurants présentent des plats qui associent plusieurs traditions culinaires.

Avec À Nous deux Paris!, J.P. Nishi réussit à reconstituer son expérience d'expatrié japonais en organisant pour sa part les épisodes d'un récit centré sur son travail dans une épicerie japonaise et sur son activité en tant que jeune auteur de mangas. Il s'agit cette fois plus franchement d'une description des interactions heureuses ou malheureuses avec la société française sans insister forcément sur la gastronomie, celle-ci étant au-dessus de ses moyens. Néanmoins, par son travail, le héros de J.P. Nishi est associé à l'univers décrit dans le guide précédent (la communauté japonaise à Paris), et il doit souvent répondre aux interrogations des Français sur les produits alimentaires japonais. Il est notamment confronté à la demande continuelle des clients français qui réclament une sauce soja sucrée, celle qui est servie dans tous les restaurants de sushis tenus par des cuisiniers chinois, et qu'il est bien incapable de leur fournir. Il leur vend alors de la sauce pour les brochettes de poulet à la place (Nishi, A nous deux 77). Une rubrique «manger pas cher » lui permet de dessiner le traiteur chinois et le kebab, tandis que son unique flirt, Fabienne, exige qu'il lui fabrique des onigiri, symbole pour elle de la culture japonaise (Nishi, A nous deux 151). Le dessinateur confesse qu'il n'a rien trouvé de surprenant sur son chemin à Paris, mais c'est justement cette insignifiance qu'il rend savoureuse pour les lecteurs des deux pays. Dans un second tome, Paris, le retour!, l'auteur fréquente encore la Japan Expo (salon de la culture manga) et y rencontre Christopher, directeur d'Atsuatsu, et fondateur d'Okotako, où il anticipe, en plein boom du sushi en France dans les années 90, sur d'autres spécialités moins connues : les okonomiyaki et les takoyaki. Certes, le prix est élevé, mais deux produits sont vendus, « coquille Saint-Jacques et tarama » et « 'spicy' crevettes sauce kimchi », ce qui est révolutionnaire pour ces boulettes de poulpe en beignets.

L'intérêt de ces différents guides ou mangas, de ces témoignages, comme celui de Fujimoto, de Delarcourcelle ou de Feneau, est d'allier une approche précise, professionnelle souvent, parfois servie copieusement par l'illustration photographique ou graphique, à un regard décalé et très personnel. Le but de ces ouvrages est certainement de mettre un contenu précis sur des notions trop connues (le règne du poisson cru, le sushi, le saké) ou trop mystérieuses (la cuisine kaiseki par exemple). Un autre objectif est de démontrer combien certains aspects de la gastronomie et de la culture populaire du Japon restent encore à découvrir en France.

\section{APPROCHES PHILOSOPHIQUES DU GOÛT}

Rosanjin Kitaôji (1883-1959) est un génie de la cuisine, mais aussi un calligraphe, céramiste de renom, créateur de restaurants au concept unique : il incarne le caractère transdisciplinaire de la question du goût et des saveurs. Il est considéré comme l'inventeur de la gastronomie au Japon, bi-shoku, soit «l'esthétique du manger», ou «le beau autour de la table » (Mussard). On voit ici que la gastronomie pour le Japon relève à l'origine d'une esthétique visuelle et de l'art de présenter les plats dans un environnement esthétique réussi. Kitaôji est celui qui a élevé la cuisine au rang d'un art total. En effet, après avoir commencé sa carrière de céramiste, il ouvre une galerie d'arts de la table et cuisine pour ses clients. Ce sera l'origine de son mythique Bishoku club ou «Club des gourmets ». Par la suite, il ouvrira en 1925 un restaurant, le Hoshi ga oka saryô. Chacun d'entre eux était conçu comme un espace total où les murs étaient décorés par ses peintures et ses calligraphies, et du menu aux couverts, tout était imaginé par ses soins : le restaurant devenait ainsi un espace théâtralisé pour une cérémonie précieuse. Pour lui, la

Alternative francophone

https://journals.library.ualberta.ca/af/index.php/af 
vaisselle était l'habit de la cuisine, et il affirme dans La Voie du goût : "grave dans ton esprit qu'un monde si profane et si nécessaire, le monde du goût, existe » (Mussard). ${ }^{4}$ Sa formation de céramiste lui permet notamment de repenser la relation entre la vaisselle et les aliments sur des bases nouvelles, en s'appuyant sur différents styles de céramique du Japon ou sur les traditions chinoises et coréennes. Le chef cuisinier d'un restaurant de sushis réputé de Ginza, le Ginza kyûbei, reconnaît l'originalité de ses choix : en effet, la couleur des produits de saison doit être mise en valeur par la vaisselle. Pour cela, les céramiques simples, comme celles de Bizen, de Shino ou Oribe, peuvent faire sentir leur matière sans nuire au plat lui-même, mais les œuvres de Kitaôji sont beaucoup plus dynamiques, et c'est un véritable défi pour le maître sushi que d'utiliser sa vaisselle. Kitaôji privilégiait une cuisine proche de la nature, inspirée par ses beautés, et son mot d'ordre était «manger nature », autrement dit s'accorder avec les saisons et les mettre en valeur. En effet, selon lui, si la matière première de la cuisine est la nature, tout comme est naturel le désir de manger, il entendait élever ces éléments au rang d'un art. Cet homme raffiné entendait donc sublimer l'opposition entre nature et culture, et faire d'une faiblesse initiale un atout. Par exemple en utilisant une combinaison de différents styles de poterie épurés pour accompagner au mieux des aliments cuisinés au plus près de leurs qualités originelles. Il défendait par ailleurs l'idée que tout était utilisable dans un produit naturel, tout comme une céramique défectueuse pouvait être retravaillée ou valorisée. Ces idées sont très modernes, et proches de certaines conceptions contemporaines en France. ${ }^{5}$ L'importance de ce personnage est telle qu'il est devenu une figure mythique aux biographies contrastées en raison de ses écarts de conduite et de son approche paradoxale des BeauxArts. Il a inspiré à Tetsu Kariya et Akira Hanasaki un manga culinaire à partir de 1983, Oishinbo (Le Gourmet), qui lui rend hommage et dont cent millions d'exemplaires ont déjà été écoulés. Un personnage de ce manga, Yûzan Kaibara, est directement inspiré par Rosanjin Kitaôji. La vie et l'œuvre de Rosanjin Kitaôji alimentent l'imaginaire des Japonais et sont admirées dans les autres pays. Si sa relation à la culture française est peu évidente, il a néanmoins voyagé en France en 1954, attentif au domaine culinaire et à celui de la céramique. Ce gastronome anticonformiste est un précurseur de la cuisine fusion. Ainsi, un bref texte, «Sukiyaki et canard, brève impression de la cuisine occidentale » (Arashiyama et al. 75), relate son approche inventive de la grande tradition française lorsqu'il dîne au célèbre restaurant parisien de la Tour d'argent. La traductrice de ce texte remarque qu'il est «"l'homme de lettres" dans toute sa splendeur, céramiste, calligraphe, peintre, artiste de laque, écrivain, gastronome excellent cuisinier...» (Arashiyama et al. 81), c'est-à-dire au sens d'un lettré chinois, mais non en général au sens d'un homme de lettres français. Il plie la recette du canard propre à cet établissement mythique au goût japonais. En effet, il refuse la sauce française, élément de prestige, pour ne retenir que le mode de cuisson qui convient parfaitement à ses desseins. Cette cuisson naturelle effectuée, le canard est montré aux convives puis il est censé revenir en cuisine pour être nappé de sauce. Mais Kitaôji exige que le canard reste sur la table et il prépare un assaisonnement typiquement japonais avec une poche de sauce de soja et une autre de wasabi,

\footnotetext{
${ }^{4}$ À l'occasion d'une exposition consacrée au célèbre gastronome, Muriel Mussard cite la traduction d'un ouvrage célèbre au Japon de Rosanjin Kitaôji sur le site du Musée Guimet. L'exposition eut lieu du 3 juillet au 9 septembre 2013.

${ }^{5}$ En 2014, le magazine culinaire Cuisine naturelle, qui défend la saveur, les plats végétariens, et la tendance «bio », prend pour slogan : «cuisinons savoureux, sain et éthique ». Par ailleurs, la gastronomie moléculaire propose en second objectif de développer la composante artistique de la cuisine. Mais ce sont surtout les idées appliquées dans la «nouvelle cuisine », suivant le terme lancé en 1973 par deux critiques, Henri Gault et Christian Millau, qui semblent proches de celles de Kitaôji. C'est une cuisine pas grasse, ni prétentieuse, sans trucages de présentation, simple et légère, et qui sait utiliser des produits frais (cuisine de marché). Sont bannis les fonds, sauces ou mets préparés à l'avance, puis réchauffés. Elle s'oppose aux traditions immuables, comme le « canard au sang » servi à La Tour d'argent. C'est une cuisine qui veut stimuler les cinq sens et sortir des dogmes, un esprit de liberté qui était aussi celui de Kitaôji.
} 
de bonne origine, sous l'œil ébahi du maître d'hôtel et des serveurs. Ce faisant, il accomplit un raccourci saisissant entre deux cultures avec un résultat intéressant, semble-t-il, même s'il tient ensuite à goûter ce qui reste du canard sans soja ni wasabi, conformément à la recette illustre du célèbre restaurant.

Le prisme culinaire en littérature est un bon angle pour décrire les sociétés en mutation du $\mathrm{XX}^{\mathrm{e}}$ siècle, par le réalisme appliqué éventuellement à un monde considéré comme décadent (et c'est une perspective conservatrice puisque l'excès dans la recherche des plaisirs physiques est toujours condamné chez les romanciers naturalistes en particulier), ou au contraire dans l'effervescence joyeuse et légèrement hédoniste d'une aventure des plaisirs, en développant le palais par association des cultures. C'est la perspective adoptée par le romancier Jun'ichirô Tanizaki dans ses premières nouvelles et dans ses premiers romans. La littérature peut ainsi rejoindre une forme de journalisme narrativisé, qui permet d'entrer dans les mondes les plus fermés, celui des plaisirs réservés à une élite palatale. Comme le suggère Jun'ichirô Tanizaki dans « Le Club des gourmets » (Arashiyama et al. 145), l'obstination du comte, qui est aussi le président de ce club, à entrer dans ce restaurant chinois qui lui inspirera la réforme de sa propre institution, est payante; il attend à la porte des plaisirs avec patience. Il est finalement autorisé à assister (mais non à participer) aux agapes secrètes de ces dandys des baguettes. Sa démarche n'est-elle pas typique du journalisme pratiqué à des fins littéraires? C'est une véritable enquête, et une quête d'absolu, qui est menée dans la capitale japonaise pour saisir les expériences culinaires les plus innovantes. Toutefois, le but n'est pas d'aboutir à un guide ouvert à tous, comme le guide Michelin, mais de permettre une consommation sélective et éphémère à l'usage d'un club secret et fermé, que les considérations financières ne freinent jamais.

Sans avoir eu connaissance du récit de Tanizaki, «Le Club des gourmets », j’avais développé sous le titre «Le Gastronome », et comme le dénouement d'une série d'enquêtes policières, le cas d'un personnage fantasque, décédé suite à l'ingestion imprudente de fugu en sashimi (Kober). ${ }^{6}$ L'approche de la gastronomie comme une passion fatale et excessive y était très présente, exactement comme chez le romancier japonais. Ce récit s'appuyait sur des faits journalistiques, mais aussi sur l'expérience de quelques restaurants entre 1996 et 1999 dans la capitale du Chûbû, Nagoya. Le récit commence par l'évocation de l'anti-gastronomie, le hamburger de chez Mc Donald's. L'offre est très variée, avec des adaptations au goût national parfois surprenantes, en utilisant algues séchées, wasabi et sauce de soja. À une échelle plus prestigieuse, les transferts culturels aboutissent à la fusion des idées chez les grands chefs étoilés de Paris ou de Tokyo. Pour revenir à l'exotisme à l'envers que représente la culture américaine, le cas de Mc Donald's est un bon exemple aussi bien que toutes les chaînes de style occidental (Doutor, Donuts, KFC...) bien implantées dans les centres villes, les galeries commerciales et en périphérie, sur les routes nationales où s'égrènent les drive et les take away. Il existe un engouement et une sociologie particulière du consommateur japonais, un écart générationnel, car ce sont les personnes d'âge mûr qui sont en général mieux informées de l'existence d'une cuisine locale ou régionale. Le journaliste Jake Adelstein évoque ce paradoxe d'une ouverture totale du goût, à propos des policiers du koban (ilôtage de quartier), pour obtenir des informations : «J'apportais des donuts, ce qui à mon avis ne faisait pas partie du régime alimentaire habituel des flics du Japon, mais ça n'avait pas l'air de les gêner. En fait, ils les aimaient peut-être aussi pour cette raison » (76). Le local est certainement en voie de régression face à la globalisation. Le Japon offre une forme de résistance à l'uniformisation du goût qui n'est pas

${ }^{6}$ Ce récit est le dernier d'un ensemble de cinq récits réunis en 2000 sous le titre L'Archipel des osselets.

Alternative francophone

https://journals.library.ualberta.ca/af/index.php/af 
incompatible avec un lâcher-prise des restaurants du type Beer parlour, dans un style de kermesse bavaroise où les plats les plus incompatibles peuvent être servis ensemble, avec un niveau de qualité très bas. On se situe à l'opposé de la tradition qui veut que les établissements servent un seul type de plat, quitte à ménager un aspect ludique et varié (le sushi tournant, ou kaitenzushi, où les soucoupes garnies de sushis tournent sur un mini-tapis roulant). Dans ce récit, « Le Gastronome », j'avais choisi d'opposer, d'une manière assez visible, le goût mondialisé à certaines traditions locales, voire à une pratique marginale, comme la consommation intégrale du fugu (poisson-lune), c'est-à-dire du foie toxique de ce poisson $^{7}$, qui peut figurer au menu de restaurants élégants aussi bien que de ceux que l'on peut rencontrer dans les quartiers déclassés, comme celui de Tsutenkaku, au sud d'Osaka. Une vieille tour métallique domine ce quartier resté dans le jus des années 60 , où l'on trouve une atmosphère marginale, jusque dans l'intitulé des menus et de spécialités étranges comme les brochettes de crocodile, le fugu et la soupe de tortue à carapace molle (suppon). Ces restaurants jouxtent les salles de jeu de mah-jong ou de vieilles salles de cinéma au programme composé de films de yakuzas en continu. Avec les yakitori (brochettes de poulet), les sushis sont devenus un article d'exportation, et un marqueur aussi évident pour le Japon que la pizza pour l'Italie, mais les filets de fugu représentent un must, peu connu quoique souvent mentionné comme un paradoxe japonais. L'évoquer dans une fiction était peut-être une forme de dérision d'un contenu essentialisé de la culture nipponne, tout comme peut l'être le wasabi dans le film français du même nom. J'avais imaginé un personnage de gastronome qui rendait hommage par son nom, Rosanjin Numata, au véritable Rosanjin Kitaôji. Le fugu est lié à un aspect souvent évoqué à propos du Japon, la mort volontaire, et le souci perfectionniste d'aller jusqu'au bout d'un apprentissage. En cela, le personnage du gastronome était une figure intéressante pour donner une tonalité humoristique à un volume centré sur la criminalité. En effet, le Japon, à l'époque où je rédigeais des notes pour ce recueil d'enquêtes policières, était pris dans une spirale de faits divers sanglants. Y figurait également le crime par empoisonnement sous un jour crapuleux, mais la mort accidentelle, par excès de gourmandise, avait une coloration moins sombre. L'excès gastronomique mène tout droit à la mort, comme le soutient également Tanizaki dans «Le Club des gourmets ». C'est une course à l'abîme sauf que, dans la tradition historique française, et même parfois dans l'actualité de certains chefs étoilés, ce sont plutôt les cuisiniers qui se donnent la mort. Il semblerait que la méticulosité et le lent apprentissage, voire une transmission de génération en génération, la considération absolue d'une équipe pour son chef, rendent l'échec plus rare au Japon.

La culture gastronomique au Japon atteint un absolu lorsqu'elle excelle à stimuler les cinq sens, touchant à une variété de disciplines artistiques autour de l'art purement culinaire. La fascination littéraire entre à son tour en jeu lorsque des écrivains s'interrogent sur le sens de la recherche dans le domaine du goût, et

\footnotetext{
${ }^{7}$ Le fugu (tetraodontidae) est un poisson dont certains organes contiennent de la tetrodotoxine, un neurotoxique sans antidote connu, qui paralyse le système nerveux, provoque un arrêt respiratoire, et éventuellement la mort, en une journée. L'effet du poison est analogue à celui du cyanure. Il engourdit d'abord la bouche, et provoque des picotements sur les lèvres au contact d'une goutte infinitésimale de poison, ce qui ajoute au plaisir de la dégustation. C'est néanmoins un mets très populaire au Japon, et sa chair sucrée est recherchée, pour un prix exorbitant. Il est interdit de le vendre entier, et seul un chef fugu ayant obtenu une licence très sélective, au bout de cinq années d'entraînement, est habilité à retirer les parties dangereuses qui peuvent être les ovaires, le foie, la peau ou même la chair. Il faut aussi savoir distinguer les espèces autorisées à la vente. Ce poisson d'hiver est consommé en sashimi (et ce sont de magnifiques tranches fines comme du papier, dont la blancheur est rehaussée par un saupoudrage de paillettes d'or, et qui forment des rosaces ou d'autres figures élégantes) ou en soupe (Nabe). En 2008, à Wakayama, cinq Japonais d'un âge situé entre cinquante et soixante ans sont tombés malades après avoir commandé un menu spécial qui comportait du foie de fugu. Le picotement provoqué sur les lèvres survient si l'on consomme des parties proches du foie ou des ovaires. Visiblement, certains Japonais se délectent à l'idée de ressentir cette sensation qui peut être un prélude à la mort.
} 
lui donnent pour aboutissement une forme de mort volontaire, qui a souvent été associée à la culture japonaise.

\section{GRAPHISMES ET APPORTS VISUELS}

J'ai déjà évoqué l'importance d'une association entre graphisme, photographie et texte afin de caractériser plus fortement la gastronomie japonaise dans l'esprit des lecteurs. Deux exemples montrent ici comment l'évocation de la cuisine japonaise peut s'approprier des styles graphiques et des esthétiques bien différentes, voire opposées.

Les dessins de Nicolas de Crécy accompagnent Esthétiques du quotidien au Japon avec des dessins réalisés à la villa Kujoyama de Kyoto en 2008 et la couverture montre une table de restaurant traditionnel. Leur élégance graphique associée à l'aquarelle sourde s'accorde bien à l'esthétique du wabi sabi japonais, avec des couleurs shibui, un peu ternes et passées. Comme le remarque un géographe à propos de la gastronomie japonaise, « dans ce type de cuisine, la présentation visuelle importe autant que la finesse du goût. Un repas de kaiseki s'apprécie avec les cinq sens et en particulier la vue » (Baumert 172). Une seconde remarque porte sur le paradoxe du guide Michelin 2008 pour Tokyo. En effet, s'il montre l'ouverture des possibles, ce guide se heurte à la nature secrète de la gastronomie japonaise : «Car le secret et l'importance accordée aux habitués sont des caractéristiques essentielles du raffinement en matière de gastronomie » (183). Nicolas de Crécy semble avoir parfaitement intégré cette idée d'élégance discrète codifiée au fil de l'histoire du Japon, non sans parfois s'octroyer l'écart de quelques monstrueux démons traversant à grandes enjambées la ville classique par excellence, Kyoto.

On ne pourrait imaginer plus grand contraste en évoquant maintenant un manga qui privilégie les espaces ordinaires, clinquants, et populaires qui orientent l'attention vers un Japon quotidien en principe exclu de toute approche culturelle par son manque de raffinement apparent. Le personnage imaginé par Masayuki Kusume et par Jirô Taniguchi, « le gourmet », est remarquable par son absence d'a priori et par son caractère aventureux. Le mot « gourmet » est ici directement calqué sur le mot français grâce au syllabaire katakana. En tant que représentant de commerce en prêt-à-porter, il est amené à se déplacer partout en ville, et donc à manger dans divers quartiers. Il est un amateur exigeant et observe avec attention aussi bien les plats, le goût que le personnel du restaurant et la clientèle, ses habitudes. Cet ouvrage est un manga, c'est-à-dire beaucoup plus qu'une simple information dessinée au graphisme clair et précis aux trois couleurs comme on le voit mis en œuvre dans la section « cuisine » d'un guide du Japan Traveler Bureau. On n'y trouve pas les ingrédients à ajouter pour composer un kitsune udon ni leurs définitions entre parenthèses, ou bien les différents types de restaurants avec leurs enseignes et leur disposition matérielle spécifique. Ce n'est pas une initiation au sens encyclopédique, sans immersion, où la recherche esthétique le cède au souci de précision. Le seul point commun avec une page dessinée de guide touristique serait la ligne claire de l'école belge transposée dans un dispositif de manga. On trouve des contours bien dessinés, des personnages et des décors minutieusement restitués pour ce qui est autant une déambulation urbaine qu'un menu de restaurant. En effet, les dix-huit chapitres du sommaire renvoient à autant de plats commandés ou de lieux visités. Chaque chapitre porte en frontispice avec le titre une image du plat consommé, comme cette assiette de porc sauté et riz à San'ya, Tokyo. Il ne faut pas oublier que les cires ou figurines en plastique sont omniprésentes dans les vitrines des restaurants japonais, et que ce fut à l'ère Meiji que l'habitude fut prise de modeler dans la cire les plats occidentaux tant ils étaient incompréhensibles pour les Japonais de l'époque. Le manga reproduit la table avec le détail

Alternative francophone

https://journals.library.ualberta.ca/af/index.php/af 
des commandes, les prix, les menus et le dialogue de la commande. Le dessin est très précis et réaliste, et le contenu des assiettes, tous les ingrédients, sont détaillés, par exemple les sept éléments d'un unagi-don, ou bien tous les éléments d'une dînette de bureau achetés au konbini (convenience store). Le traitement narratif et graphique sublime ces repas souvent simples, et le lecteur peut s'identifier à cette expérience de « banalyse ». ${ }^{8}$ Il suit pas à pas ce reporter acharné pour qui la découverte des plaisirs du goût semblent remonter à une histoire intime. La dimension autobiographique domine de façon encore plus explicite dans l'ouvrage de Julie Blanchin Fujita, J'aime le nattô, où le dessin amateur s'associe à un texte bilingue sur papier quadrillé imitant le carnet intime. C'est le journal d'une expatriée qui prend le nattô (des graines de soja fermentées qui accompagnent souvent le riz nature) pour un sésame de la cuisine familiale, avec une page pour en faire l'éloge. Les dessins personnalisés des ingrédients sont accompagnés de leurs noms en deux langues et de leur description, tout comme les plats principaux ou des spécialités locales. L'auteure, avec cette mosaïque de textes et de graphismes, nous immerge dans son expérience sensible d'expatriée, avec une écriture qui épouse les contours des dessins. Cela donne des pages très denses, ce qui est un trait du graphisme japonais quand il veut expliquer une recette ou un itinéraire. Le dessin reste amateur, mais avec un style personnel qui est déjà une interprétation poétique du réel.

Ces trois auteurs de livres illustrés ou de mangas partagent une expérience autobiographique, que ce soit celle d'un résident occasionnel, celle d'une expatriée temporaire ou celle d'un personnage de Japonais (qui découvre les zones interstitielles et savoureuses de la capitale et de ses environs). Ils témoignent, chacun avec des choix graphiques et esthétiques différents, du rapport intime qui s'établit entre l'être humain et les aliments consommés quotidiennement. La nourriture devient une clé efficace pour entrer dans un Japon ordinaire, mais subtil qui échappe à une saisie superficielle ou touristique.

\section{EXPRESSIONS CINÉMATOGRAPHIQUES}

Le graphisme ou la photographie exercent une séduction importante sur l'explorateur du goût. Ces moyens ont aussi une grande efficacité didactique, mais l'image animée, le cinéma (et le cinéma japonais occupe une place unique dans la cinéphilie européenne), semblent avoir un pouvoir encore plus grand. Les films feraient sentir presque physiquement l'émotion esthétique et sensorielle qui peut s'éprouver devant des mets raffinés ou savoureux.

Parmi d'autres films plus récents que l'on aurait pu évoquer, comme ceux de Hirokazu Kore-Eda, la comédie de Jûzô Itami, Tampopo (1985), paraît remarquable. Elle évoque notamment un restaurant de râmen, les nouilles chinoises en soupe, un plat très populaire, bon marché, élevé ici au rang d'un art. Tampopo, la jeune veuve qui aspire à maîtriser l'art de la nouille espionne les concurrents, essaye de comprendre comment préparer un bouillon. Le meilleur est un vieux monsieur déclassé nommé « le professeur » qui vit parmi des clochards gastronomes illuminés. On découvre ainsi l'excellence qui peut exister dans un domaine pourtant modeste à partir d'une lente formation. Ce film a le mérite de faire découvrir au public français autre chose que l'élégante cuisine kaiseki, qui, au fond, concerne très peu de Japonais. Mais ce film va beaucoup plus loin en explorant le lien entre amour, mort et gastronomie. En effet, le plaisir gustatif accompagne jusqu'à la fin certains personnages, comme ce père de famille qui se précipite pour manger le dernier repas préparé par son épouse qui tombe dans le coma, ou bien un

\footnotetext{
${ }^{8}$ C'est un mouvement critique et expérimental né en France dans les années 80 en réaction notamment à l'exigence de rentabilisation du temps et de marchandisation du monde. Des congrès se sont tenus pendant plus de dix ans dans un lieu insignifiant. Le gourmet, qui est un représentant de commerce, se plaît à découvrir pendant ses pauses des lieux ordinaires et savoure le plus souvent des plats très bon marché ou des spécialités populaires.
} 
gangster gourmand qui pense en mourant à ce qu'il pourrait manger de bon. De la naissance à la mort, l'être humain est sensible aux plaisirs de bouche. Par ailleurs, ce film montre bien l'universalité du plaisir gastronomique qui dépasse les clivages sociaux ou culturels. Ainsi, un riche rentier, un gangster, des gens pauvres, une pêcheuse d'ormeaux peuvent communier dans le même goût. Le plaisir gastronomique relie entre elles les différentes catégories sociales. Enfin, ce film propose quelques interactions culturelles amusantes, qui réduisent la valeur des « bonnes manières » ou relativisent les codes sociaux, comme celui d'aspirer les spaghettis ou les nouilles en faisant du bruit ou non. La manière dont la gastronomie européenne peut être admirablement servie au Japon est dite tout autant que l'incompréhension qu'elle suscite en général. Ce film montre admirablement tout le charme et tout le plaisir qui peuvent être retirés de la fréquentation d'un endroit aussi banal qu'un petit restaurant de râmen, et il explique parfaitement comment les préparer avec talent, ce que font par ailleurs, sur un mode purement documentaire, d'innombrables blogues et sites de cuisine japonaise ou asiatique.

Mentionnons enfin Wasabi, le film d'action de Gérald Krawczyk, sorti en 2001. Celui-ci n'est pas centré sur la cuisine, mais on y trouve au moins deux allusions liées à la maladresse et au comportement viril de l'inspecteur Hubert, parti sur les traces de son ancienne fiancée, Miko. Il boit directement le cruchon de saké qui lui est servi, sans égard pour les règles du savoir-vivre, et le vide devant ses hôtes médusés. Ensuite, vers la fin du film, il mange avec ses doigts la pâte verte de moutarde de raifort, dite wasabi, comme si c'était un dessert délicieux, et non un des condiments du sashimi à utiliser avec parcimonie. Ce film popularise encore l'association entre poisson cru et Japon à travers cette moutarde verte de wasabi, présente sur la table de tous les restaurants dits « japonais » de la planète. Moins inventif que Rôsanjin Kitaôji, le héros indique sur le mode humoristique, une incompatibilité de goût entre deux cultures, mais au fond un gigantesque appétit pour l'étrangeté des saveurs.

Finalement, l'art cinématographique, comme les autres arts évoqués plus haut, peut aussi bien produire une vision pauvre et polarisée sur des idées reçues que le déploiement d'une richesse insoupçonnée au creux des apparences. L'intrigue culinaire permet de tisser la complexité des relations humaines au sein d'un microcosme social, ou le survol d'une culture réduite à quelques archétypes.

Plus que d'autres domaines sans doute, celui du goût appelle une immersion personnelle de l'écrivain ou du journaliste, et un traitement original de la documentation, comme si la dimension visuelle de la gastronomie japonaise appelait naturellement la description littéraire, l'image filmique ou le graphisme. Il n'est pas dit d'avance que le travail de l'imagination à partir des mots de l'écrivain soit moins concluant que la perception des images fixes ou mobiles et du graphisme des dessinateurs. Dans le même temps, cette gastronomie doit rester, selon certains avis autorisés, presque secrète parce qu'elle est conçue pour un nombre limité de clients, faute de diminuer en qualité. Tout se passe comme si la haute gastronomie japonaise - et même la gastronomie de quartier - était réservée aux happy few. Une bonne adresse perd vite de sa qualité à être trop sollicitée. Néanmoins, les cultures française et japonaise s'apparient souvent bien sur le thème des plaisirs gustatifs perçus comme une voie d'accès à un plus grand raffinement humain. Il faut parier sur un nouvel éclairage de la voie du goût avec pour objectif une meilleure connaissance de la culture japonaise.

Alternative francophone

https://journals.library.ualberta.ca/af/index.php/af 


\section{OUVRAGES CITÉS}

Arashiyama, Kôzaburô et al. Le Club des Gourmets et autres cuisines japonaises. P.O.L., 2013.

Adelstein, Jake. Tokyo Vice. Traduit par Cyril Gay, Marchialy, 2016.

Baumert, Nicolas. « Haute gastronomie et produits de qualité. Le luxe alimentaire japonais à la croisée des traditions. » Esthétiques du quotidien au Japon, dirigé par Jean-Marie Bouissou, IFM/Regard, 2010.

Binet, Laurent. « Gonzo, vous avez dit gonzo ? » Marianne, www.marianne.net/culture/gonzo-vous-avezdit-gonzo. Consulté le 9 avril 2020.

Blanchin Fujita, Julie. J'aime le nattô - une aventure au Japon. Hikari, 2018.

Delacourcelle, Philippe. Le Goût du Japon - itinéraire d'un cuisinier français au pays du Soleil-Levant. Jean-Paul Rocher, 2009.

Dubois, Jean-Pierre. « Des écrivains français à la découverte du Japon. » France-Japon - Une nouvelle histoire, Revue des deux mondes, avril 2013.

Feneau, Laurent. Sakés. Argol, 2013.

Fujimoto, Kenji. Le Cuisinier du dictateur. Traduit par Stéphanie Nagai, Hugo Doc, 2019.

Gauntner, John. The Saké Handbook. Tuttle, 2002.

Harper, Philip. The Insider's guide to sake. Kodansha international, 1998.

Hanasaki, Akira et Tetsu Kariya. Oishinbo (le Gourmet). Shôgakukan, 1983-2014.

Kaganski, Serge. «Philippe Garnier raconte le "nouveau" journalisme. » Les Inrockuptibles, www.lesinrocks.com/2009/05/18/actualite/actualite/philippe-garnier-raconte-le-nouveaujournalisme/. Consulté le 08 avril 20020.

Kober, Marc. L'Archipel des osselets. Fayard, 2000.

Mussard, Muriel. «L’Art de Rosanjin, génie de la culture française. » Musée Guimet, 2013, https://www.guimet.fr/sites/saison-japonaise/l-art-de-rosanjin.html. Consulté le 04 avril 2020.

Nishi, J.P. A nous deux, Paris !. Philippe Picquier, 2011.

Nishi, J.P, Paris, le retour !. Philippe Picquier, 2012.

Norimatsu, Minako. Guide du Japon à Paris. Chêne, 2013.

Pons, Philippe et Pierre-François Souyri. Le Japon des Japonais. « L'autre guide », Liana Levi, 2002, réédité en 2007.

Sekiguchi, Ryoko. Nagori. P.O. L, 2018.

Tampopo. Écrit et réalisé par Jûzô Itami, joué par Tsutomu Yamazaki, et al., Itami et New Century productions, 1985.

Taniguchi, Jirô et Masayuki Kusumi. Le Gourmet solitaire. Traduit par Patrick Honnoré, Sahé Cibot, Casterman, 2005.

Wasabi. Réalisé par Gérard Krawczyk, écrit et produit par Luc Besson, joué par Jean Reno, et al., Europacorp/TF1, 2001. 
Woo, Elaine. « Bil Cardoso, 68, writer introduced 'gonzo'. » Los Angeles times, https://www.latimes.com/archives/la-xpm-2006-mar-12-me-cardoso12-story.html. Consulté le 9 avril 2020. 